\title{
Modification of meta-film
} surface impedance expression for accurate scattering analysis of meta-surface using equivalent transmission line
model

\author{
Akie Kuriyama, Toru Uno, and Takuji Arima ${ }^{\text {a) }}$ \\ Graduate School \& Engineering, Tokyo University of Agriculture and Technology, \\ Naka-cho, Koganei-shi, Tokyo 184-8588, Japan
}

a)t-arima@cc.tuat.ac.jp

Abstract: Scattering properties of meta-surfaces for plane wave incidences can successfully be analyzed using their own transmission line models (TLMs). The main idea of the TLM is the decomposition of a periodic structure into a frequency selective surface (FSS) or a meta-film and dielectric slab as a spacing medium between the FSS and a ground plane. Hence, how precisely obtain the scattering property of the FSS is one of essential points. This paper modifies a surface impedance expression of the FSS composed of square patch array so as to enable us to apply for general case, and incorporates it into the equivalent transmission line model of the metasurface. The effectiveness of the proposed model is numerically confirmed by comparing with the FDTD calculation.

Keywords: meta-surface, meta-film, transmission line model, FDTD method

Classification: Antennas and Propagation

\section{References}

[1] F. Capolino, ed., Metamaterials Handbook, Part I \& Il, CRC Press, 2009.

[2] C. Caloz and T. 1toh, Electromagnetic Metamaterials: Transmission Line Theory and Microwave Applications, John Wiley \& Sons Inc., 2006.

[3] Y. Yang and Y. Rahmat-Samiii, Electromagnetic Band Gap Structures in Antenna Engineering, Cambridge Univ. Press, 2009.

[4] C. L. Holloway, M. A. Mohamed, E. F. Kuester, and A. Dienstfrey, "Reflection and transmission properties of a metafilm: With an application to a controllable surface composed of resonant particles," IEEE Trans. Electromagn. Compat., vol. 47, no. 4, pp. 853-865, Nov. 2005. DOI:10.1109/TEMC.2005.853719

[5] A. Kuriyama, T. Uno, and T. Arima, "Application of meta-film surface impedance to equivalent transmission line model of meta-surface for scattering 
analysis," Proc. International Symposium on Antenna and Propagation 2016 (ISAP2016), pp. 326-327, October, 2016.

[6] J. F. Douglas and E. J. Garboczi, "Intrinsic velocity and the polarizability of particles having a wide range of shapes," in Advances in Chemical Physics, ed. I. Prigogine and S. A. Rice, John Wiley \& Sons, Inc., vol. 91, pp. 85-153, 1995.

[7] S. Tretyakov, Analytical Modelling in Applied Electromagnetics, Artech House Inc, 2003.

\section{Introduction}

Many kinds of meta-surfaces including an electromagnetic band gap (EBG) structure have been developed in the fields of antennas and propagation, microwave and optical devices and electromagnetic compatibilities [1]. Electromagnetic properties of the meta-surface for both a scattering of plane wave and a dispersion diagram of surface waves are often analyzed by the transmission line model, and by numerical techniques such as the finite difference time domain (FDTD) method when more accurate results are needed $[1,2,3]$.

In the case of scattering analysis using the TLM discussed in this paper, the meta-surface is often modeled by the sum of two composite parts. One is the part of planar meta-film, other the dielectric slab between the meta-film and the ground plane. In ref. [4], transmission line modeling method of meta-film was proposed. On the other hand, this letter proposes high accuracy modeling method of the transmission line for meta-surface which was backed by perfect electric conductor (PEC). Hence, the modeling of the meta-film influences the entire accuracy as a result. Recently, the reflection and transmission properties of the meta-film have been investigated comprehensively, and the accurate surface impedance expression of square patch array has been derived both for TE and TM incident plane waves under the condition that the gap between the patches is very small compared with the period. We modify this surface impedance expression so as to remove this limitation, and incorporate it into the equivalent transmission line model of the meta-surface composed of the square patch array on the dielectric substrate backed by a PEC [5]. However, the effectiveness of the modified method was not clearly shown in ref. [5], because, the result was shown only one angle. In this paper, the effectiveness of the method is carefully confirmed by comparing multi angels. The accuracy of the proposed method is numerically tested by comparing with the FDTD calculation.

\section{Modification of surface impedance of meta-film and equivalent transmission line model}

The geometry of the meta-surface considered here is shown in Fig. 1. The metasurface is composed by the square patch array and the dielectric substrate backed by the PEC. The period of the patches is $T$, and the gap is $w$ as shown in Fig. 1(b). The thickness and the relative permittivity of the substrate are $h$ and $\varepsilon_{r}$, respectively. The plane wave incidences obliquely to the meta-surface with the incident angle $\theta$ (TE polarization in Fig. 1(b)). 
Considering that the patch array is placed on a dielectric interface, the original surface impedance expressions of the meta-film in the vacuum [4] are modified as follows.

$$
Z_{g c}^{T E}=-j \frac{\eta_{e f f}}{2 \alpha\left(1-\frac{k_{0}^{2}}{k_{e f f}{ }^{2}} \frac{\sin ^{2} \theta}{2}\right)}, \quad Z_{g c}^{T M}=-j \frac{\eta_{e f f}}{2 \alpha}
$$

where $\alpha$ is given by

$$
\alpha=\frac{k_{e f f}}{2} \frac{N \alpha_{E}}{1-\frac{N \alpha_{E}}{4 R}}
$$

and where $k_{0}$ is a wavenumber in vacuum, $k_{\text {eff }}=k_{0} \sqrt{\varepsilon_{\text {eff }}}, \eta_{\text {eff }}=\eta_{0} / \sqrt{\varepsilon_{\text {eff }}}$ is an effective wave impedance, $\eta_{0}$ is the wave impedance in vacuum and $\varepsilon_{e f f}=\left(\varepsilon_{r}+1\right) / 2$. The coefficients $N$ and $R$ are given by $N=1 / T^{2}$ and $R=0.6956 T$. Another coefficient $\alpha_{E}$ is called an electric polarizability and originally given by $\alpha_{E}=1.02(T-w)^{3}$ in [6]. However, eq. (1) was derived essentially under the condition of $w \ll T$, we checked the accuracy of eq. (1) using the FDTD method. Fig. 2(a) shows the error of a phase of impedance as a function of $x$ when we put $\alpha_{E}=x(T-w)^{3}$ for a normal incidence. It is found that $x=1.15$ the best value.

Characteristic impedances of the transmission line in the dielectric substrate for TE and TM waves are given by

$$
Z_{d s}^{T E}=j \omega \mu \frac{\tan (\beta h)}{\beta}, \quad Z_{d s}^{T M}=j \omega \mu \frac{\tan (\beta h)}{\beta} \cos ^{2} \theta^{\prime}
$$

where $\beta=\sqrt{k_{0}^{2} \varepsilon_{r}-k_{0}^{2} \sin ^{2} \theta}$ is the wavenumber in the substrate [7]. $\theta^{\prime}=\arcsin \left(\sin \theta / \sqrt{\varepsilon_{r}}\right)$ is the angle of reflection calculated by the Snell's law. Using the above parameters, the equivalent transmission line model of the metasurface for scattering analysis can be illustrated as Fig. 2(b). The surface impedance of the meta-surface $Z_{s}$ is a parallel connection of the patch array impedances and the dielectric substrate impedances.

$$
Z_{s}^{-1}=Z_{g c}^{-1}+Z_{d s}^{-1}
$$

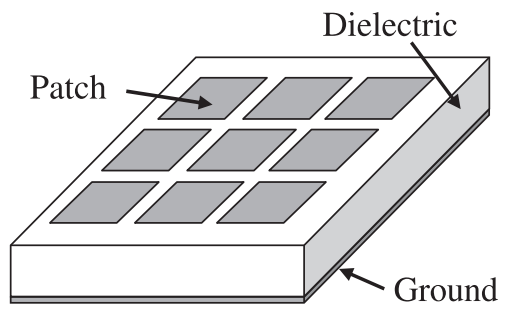

(a) Air view

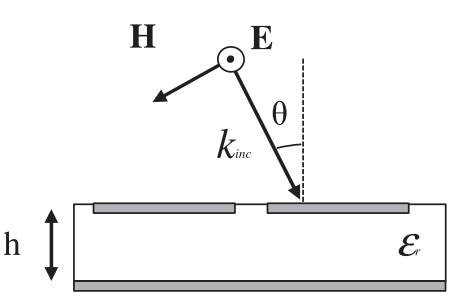

(b) Side view

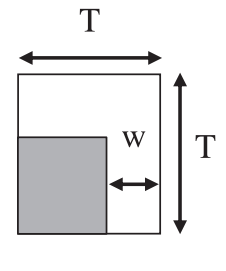

Fig. 1. Two dimensional square patch array on dielectric slab backed by PEC 


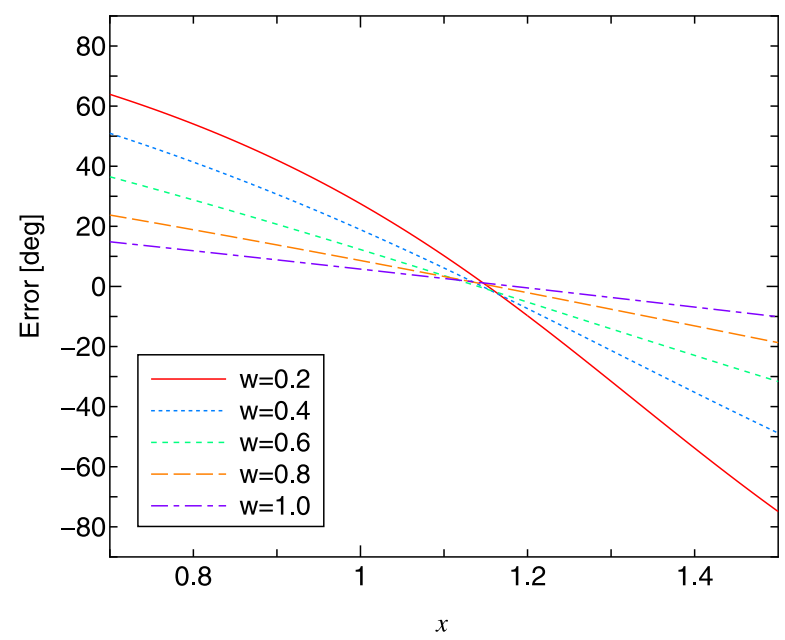

(a) Phase error of surface impedance $(T=2.0 \mathrm{~mm})$

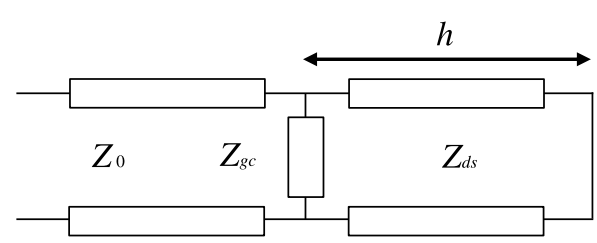

(b) Equivalent transmission line model

Fig. 2. Phase error of surface impedance and transmission line model of meta-surface

\section{Calculation results}

In order to confirm the accuracy of the proposed model, we have calculated frequency characteristics of the reflection coefficients for both TE and TM plane waves, and compared with the FDTD. In these calculations, we set $T=2 \mathrm{~mm}$ and $h=2 \mathrm{~mm}$ and $\varepsilon_{r}=4.0$. Fig. 3 shows the frequency characteristics of the phase of reflection coefficients when the gap between adjacent patches $w$ is changed. The incident angle for both polarizations is 30 and 60 degrees. It is found that the all of the results agrees excellently well with the FDTD method. Not shown here, it is noted here that the same accuracy was maintained for more large incident angle. The calculation time of the proposed model is surely negligible. 


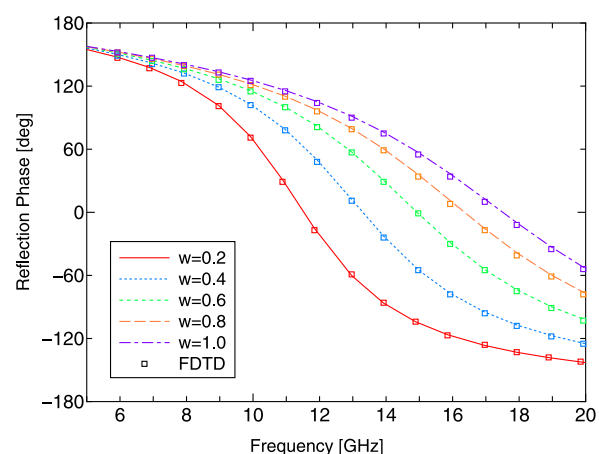

(a) TE wave, angle of incidence is $30^{\circ}$

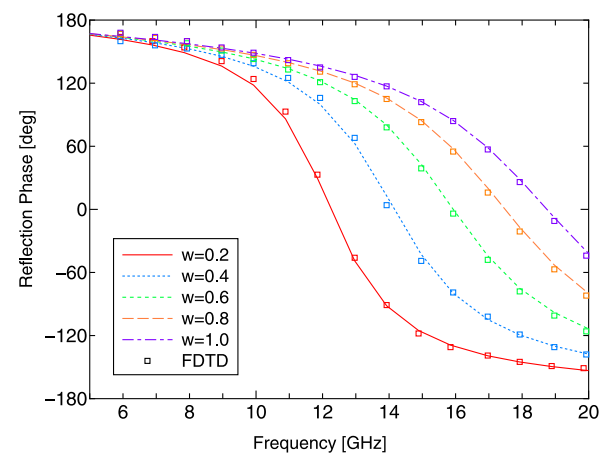

(c) TE wave, angle of incidence is $60^{\circ}$

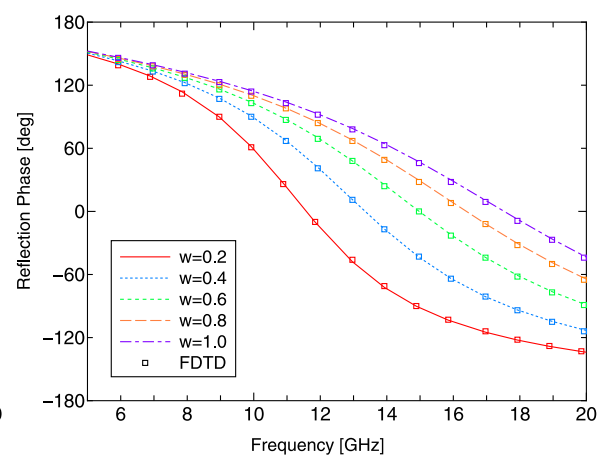

(b) TM wave, angle of incidence is $30^{\circ}$

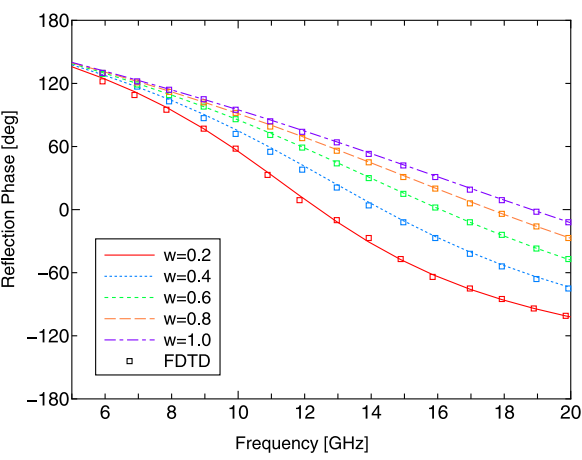

(d) TM wave, angle of incidence is $60^{\circ}$

Fig. 3. Phase of Reflection coefficient vs frequency

\section{Conclusion}

In this paper, the extremely accurate surface impedances of the periodic square patch array placed on the dielectric interface have been derived both for TE and TM plane wave incidences, and applied to the calculation of the meta-surface scattering problem. It has been demonstrated numerically that the proposed model is considerably accurate for wide frequency range. This model can also be useful for wide incident angle range from a normal incidence to a near-grazing incidence. 